Cahiers $d u$ MONDE RUSSE

\section{Cahiers du monde russe}

Russie - Empire russe - Union soviétique et États indépendants

45/3-4 | 2004

Varia

\title{
Marshall T. Poe, The Russian elite in the seventeenth century
}

André Berelowitch

\section{OpenEdition}

\section{Journals}

Édition électronique

URL : https://journals.openedition.org/monderusse/4174

DOI : 10.4000/monderusse.4174

ISSN : $1777-5388$

Éditeur

Éditions de l'EHESS

\section{Édition imprimée}

Date de publication : 1 juillet 2004

Pagination : 626-629

ISBN : 2-7132-2009-2

ISSN : $1252-6576$

Référence électronique

André Berelowitch, "Marshall T. Poe, The Russian elite in the seventeenth century », Cahiers du monde russe [En ligne], 45/3-4 | 2004, mis en ligne le 16 juin 2009, consulté le 03 septembre 2022 URL : http://journals.openedition.org/monderusse/4174; DOI : https://doi.org/10.4000/monderusse. 4174

Ce document a été généré automatiquement le 3 septembre 2022.

Tous droits réservés 


\title{
Marshall T. Poe, The Russian elite in the seventeenth century
}

\author{
André Berelowitch
}

\section{RÉFÉRENCE}

Marshall T. POE, The Russian elite in the seventeenth century. Vol. I : The Consular and ceremonial ranks of the Russian « Sovereign's Court ", 1613-1713. Vol. II : Quantitative analysis of the "Duma ranks" 1613-1713. Vammala, 2004, 469 + 283 p. (Annales Academiæ Scientiarum Fennicæ, Humaniora, 322-323)

L'ouvrage de Marshall Poe est l'aboutissement d'une enquête sur la noblesse de cour entamée il y a plus de quinze ans par l'auteur, Olga Košeleva, Russell Martin et Boris Morozov. Robert Crummey, le père spirituel de l'entreprise, avait déjà publié, en 1983, Aristocrats and servitors. The Boyar elite in Russia, 1613-1689. The Russian elite en est le prolongement, mais dans un cadre élargi, puisque l'étude est poursuivie jusqu'à l'extinction de l'ancien système des grades et qu'elle inclut désormais, outre les grades $\mathrm{du}$ Conseil, les titulaires de charges auliques, trésorier, garde du sceau, grand veneur, écuyer tranchant, etc.

L'information est puisée pour l'essentiel dans les registres (bojarskie knigi) et les rôles (bojarskie spiski) de la cour, mais comme certains de ceux-ci ont disparu, comme la plupart présentent des lacunes, il a fallu les compléter par d'autres sources : registres des rangs (razrjadnye knigi, traduit bizarrement Court deployment books), registres généalogiques (rodoslovnye knigi), divers recueils de documents et des sources secondaires, comme le précieux répertoire de S. K. Bogojavlenskij, Prikaznye sud'i XVII veka (1946).

Pour que la suite soit intelligible, il faut rappeler que les documents du xvire siècle représentent le personnel de la cour sous la forme d'un empilement de catégories. Dans l'ordre croissant des dignités, on trouve d'abord la noblesse dite de Moscou, parce qu'elle a obligation d'y résider: pages (žil'cy), gentilshommes de Moscou (moskovskie, bol'šie 
dvorjane), gentilshommes de la Chambre (strjapčie), panetiers (stol'niki) constituent les gros bataillons. Le sommet de la pyramide, collectivement désigné par "grades du Conseil » (dumnye činy), comprend les gentilshommes du Conseil (dumnye dvorjane), les secrétaires du Conseil, élite de la bureaucratie (dumnye d'jaki), les quartiers-maîtres (okol 'ničie), enfin les bojare (transcription courante : boyards).

Le premier volume de M. Poe (entièrement en russe, sauf les p.1-39) est consacré à la présentation des données. D'une part, les quatre chercheurs ont reconstitué, p. 81-382, pour chaque année de 1610-1611 à 1713, la liste des membres de la cour titulaires de charges auliques ou appartenant aux grades du Conseil. D'autre part, des fiches individuelles récapitulent le profil de carrière de tous les personnages étudiés (p. 383-469). L'ensemble, sans être absolument nouveau puisque bon nombre d'informations étaient déjà disponibles, mais dispersées dans une multitude d'ouvrages, met à la disposition des historiens un répertoire utile et commode, où les données biographiques sont complétées et précisées. On peut regretter toutefois l'omission des dates de naissance, qui ne sont connues il est vrai que pour quelques courtisans.

Le second volume, rédigé en anglais, présente beaucoup moins d'intérêt. S'appuyant sur 84 tableaux, 75 graphiques et de savants calculs de probabilités, l'auteur aboutit aux conclusions suivantes: un membre moyen de la cour n'a qu'une chance sur 30, soit un peu plus de $3 \%$, d'être promu à un grade du Conseil, à un âge qui peut varier de 25 à 45 ans, et avec des chances très réduites de promotion ultérieure. S'il est prince ou de bon lignage, ses chances de parvenir sont multipliées par cinq, par dix si en outre des membres de sa famille ont déjà goûté aux honneurs suprêmes, par quinze s'il cumule titre princier, lignage illustre et parents dans la Duma (p. 183).

C'est se donner beaucoup de mal pour enfoncer des portes ouvertes. Le sous-secrétaire transfuge Grégoire Kotošihin le disait beaucoup mieux, et plus brièvement, en 1666: « Des grandes familles d'antan, princes et bojare, beaucoup ont disparu sans retour. D'autres, aujourd'hui, leur ont succédé, dont les membres sont nommés au rang de bojare, sans jamais passer par celui de quartier-maître : ce sont les princes Čerkasskij, les princes Vorotynskij, les princes Trubeckoj [etc.]. D'autres familles, de moindre envergure, figurent parmi les quartiers-maîtres comme parmi les bojare: les princes Kurakin, les princes Dolgorukov, les Buturlin [etc.]. Les familles, enfin, dont les membres sont nommés gentilshommes du Conseil et quartiers-maittres, sont parfois d'honorable lignage, ou de noblesse moyenne, ou simples gentilshommes, mais jamais elles ne dépassent ce degré d'honneur. $»^{1}$

C'est dire on ne peut plus clairement que les nominations dépendent d'un facteur non quantifiable: la valeur d'un sang, le prestige d'une famille, l'honneur d'une lignée. En d'autres termes, le statut de chaque individu est prédéterminé par sa naissance. Les statistiques de M. Poe ne font que traduire, sans l'expliquer pour autant, ce phénomène bien connu de tous les spécialistes. Il n'y aurait encore là que demi-mal. Mais ses commentaires, les termes qu'il emploie révèlent une connaissance superficielle du sujet et risquent d'induire en erreur le lecteur non averti.

Ainsi, la hiérarchie moscovite des grades n'est pas du tout un cursus honorum, expression que l'auteur emploie constamment, mais une échelle des dignités qui servait par exemple à proportionner les indemnités en cas de procès pour injures. Le schéma qui figure dans l'ouvrage (t. I, p. 12 ; t. II, p. 24), et surtout les flèches ascendantes qui conduisent d'un grade au suivant, est particulièrement fâcheux. Il suggère un organigramme à sens unique, impression que le commentaire ne dément pas. En réalité, 
et pour ne prendre que cet exemple, les panetiers qui constituent le sommet des grades de Moscou et la pépinière des grades du Conseil, sont en majorité de très jeunes gens, et c'est en arrivant à l'âge mûr (trente ans environ) que les moins bien nés d'entre eux accèdent au grade de gentilhomme de Moscou, situé deux échelons plus bas.

Le second volet des conclusions de M. Poe concerne l'afflux dans les grades du Conseil des " hommes nouveaux" issus de lignages moins prestigieux. Cette politique, inaugurée par le tsar Alexis (1645-1676), transforme la curia regis en un corps largement honorifique, dont la fonction principale serait de distribuer la faveur monarchique à un nombre croissant de privilégiés (t. II, p. 184-185). Le phénomène n'est pas contestable, mais là encore la manière de le présenter, et plus encore les explications que l'auteur en donne, ne peuvent que susciter des réserves. Le cadre adopté pour l'enquête en est en partie responsable. Étudier les grades du Conseil sans mettre leur évolution en rapport avec celle de la cour dans son ensemble, l'observer à partir de 1613 sans se soucier de la période précédente, est un choix qui conduit presque inévitablement à des erreurs de perspective.

La croissance globale des effectifs commence dès le début du xvire siècle, puisque les courtisans, qui n'étaient que 1156 en 1589 sont déjà près de 1500 en 1604, environ 2000 en 1616, plus de 3000 en 1627, dont 839 gentilshommes choisis ${ }^{2}$, près de 4000 en 1651 sans les gentilshommes choisis. On constate donc bien une inflation des honneurs dès le milieu du siècle, même si elle n'est pas encore perceptible parmi les grades du Conseil ( $t$. I, p. 13). Les grades de Moscou étant le vivier des grades du Conseil, la croissance des premiers entraîne, presque mécaniquement, celle des seconds, mais avec un temps de retard. Du reste, la proportion entre les effectifs globaux de la cour et ceux des grades du Conseil reste étonnamment stable : environ $2 \%$ (t. II, p. 41, tableau 2A).

Cette inflation quantitative des honneurs s'accompagne d'une inflation qualitative, c'està-dire de la création de grades nouveaux, que $\mathrm{M}$. Poe passe complètement sous silence. Il est vrai qu'elle reste discrète, puisqu'elle procède par subdivision des anciens titres. Les bojare peuvent être bojare tout court, bojare « maîtres de l'Hôtel » (dvoreckie, depuis 1646) ou bojare "intimes" (bližnie, terme employé dès 1587, catégorie officielle en 1682). En 1675 apparaissent les « quartiers-maîtres intimes ». Les 886 panetiers de 1680 sont pour la plupart des «panetiers du Préau» (ploščadnye) ou «de campagne» (pohodnye); l'élite porte le titre de " panetier intime » (komnatnyj, 1623 au plus tard) ou, mieux encore, de panetier intime "de la Chambre» (spal'nik, Kotošihin, 1666). On l'aura deviné, ces nouveaux grades servent de refuge aux membres de l'aristocratie ou aux favoris du souverain, qui abandonnent le grade de panetier ordinaire à des hommes dont Boris Godunov n'aurait même pas voulu comme pages. Ils correspondent également à des nécessités techniques : le classement d'une population en augmentation constante exige la multiplication des niveaux hiérarchiques ${ }^{3}$.

Loin de moi l'idée de disqualifier les méthodes statistiques en matière de sciences humaines. Le calcul des probabilités peut se révéler précieux, au contraire, pour reconstituer des processus de décision et des choix politiques ou sociaux qui, faute de sources, échappent à l'historien. Encore faut-il les employer avec finesse, et non pas soumettre à l'épreuve des chiffres des hypothèses qui deviennent presque fausses à force d'être simplistes. 


\section{NOTES}

1. G. Kotošihin, O Rossii v carstvovanie Alekseja Mihajloviča (De la Russie sous le règne d'Alexis Mihajlovič), 4e éd., Saint-Pétersbourg, 1906, repr. La Haye-Paris, 1969, p. 23.

2. D'où la divergence avec M. Poe, t. II, p. 41, tableau $2 \mathrm{~A}$, qui donne 2130 , en excluant les gentilshommes choisis. J'arrive à un total de 2237 à partir de Bojarskaja kniga 1627 goda (Registre de la cour de 1627), Moscou, 1986.

3. Benoît Mandelbrot, Les objets fractals, 3 éd., Paris, 1989, p. 152. 